\title{
Bestimmung der Lipide im Amnion und im Fruchtwasser
}

\author{
H. Schwarzlurtner, G. Klima und W. Schmidt \\ Institut fur Histologie und Embryologie der Universität Innsbruck
}

\begin{abstract}
Zusammentassung:
Nach Anwendung von dünnschichtchromatographischen Untersuchungsmethoden konnten die im Amnion angereicherten Lipide analysiert werden. Ihr Phospholipidanteil besteht aus Lecithin. Sphingomyelin, Kephalin, Phosphatidy/serin. Phosphatidylinosit und Phosphatidyläthanol-Amin. Es handelt sich um eine Phospholipidzusammensetzung. die sich in gleicher Weise und in gleichem Verhältnis, gemessen an der L/S-Ratio, im Fruchtwasser derselben Schwangerschaft wieder nachweisen läßt.
\end{abstract}

Diese Ergebnisse werden als weiterer Beweis für die Hypothese herangezogen, daß die im Fruchtwasser suspendierten Liposomen mit den im Amnionepithel tröpfchenförmig angereicherten Lipiden identisch sind und diese nach einer Exozytose ins Fruchtwasser gelangten.

\section{Schlüsse/wörter:}

Fruchtwasser - Amnionepithel - Lipide - Dünnschichtchromatographie.

\begin{abstract}
Summary:
The lipids that accumulate in the amnion were able to be analysed with the thin-layer chromatographic method. The phospholipid content is composed of Lecithin, Sphingomyelin, Kephalin, Phosphatidy/serin, Phosphatidylinosit and Phosphatidylaethanol-Amin. It concerns a phospholipid composition that was found in the same way and in the same proportion, measured with the L/S-Ratio in the amniotic fluid of the same pregnancy. These results were taken as further proof of the hypotheses that the liposomes that are suspended in the amniotic fluid, are identical with the saturated lipid in droplet form in the amnion epithelium, and penetrate after exocytose the amniotic fluid.
\end{abstract}

Keywords:

Human amnion - amnion fluid - thin layer-chromatographic method - lipids.

Mikroskopische Untersuchungen an den Eihäuten des Menschen $(3-6)$ zeigten, daß speziell im Amnionepithel Lipidtröpfchen in großer Menge enthalten sind (Abb.1). Sie bestehen, wie histochemische Untersuchungen erkennen ließen, aus Neutralfetten und Phospholipiden. Da sich auch im Fruchtwasser Lipidtröpfchen (Liposomen) von gleicher. Gestalt und Zusammensetzung fanden (5), konnte vermutet werden, daß sie durch Exozytose aus dem Amnionepithel ins Fruchtwasser abgegeben worden seien. Nachdem in der Perinatalogie der Lecithin-Sphingomyelin-Quotient (L/S. Ratio) des Fruchtwassers als Parameter für die Bestimmung des Reifegrades der kindlichen Lunge herangezogen wird in der Annahme, die bestimmten Lipide seien mit dem Surfactant identisch, schien uns eine Überprüfung der eigenen Ergebnisse von Bedeutung. Es wurde die Hypothese aufgestellt, daß die im klinischen Test bestimmten Phospholipide nicht ausschließlich aus der Lunge des Kindes, sondern überwiegend aus dem Amnion stammen. Diese Hypothese wurde noch durch die Beobachtung bestärkt, daß die Liposomen des

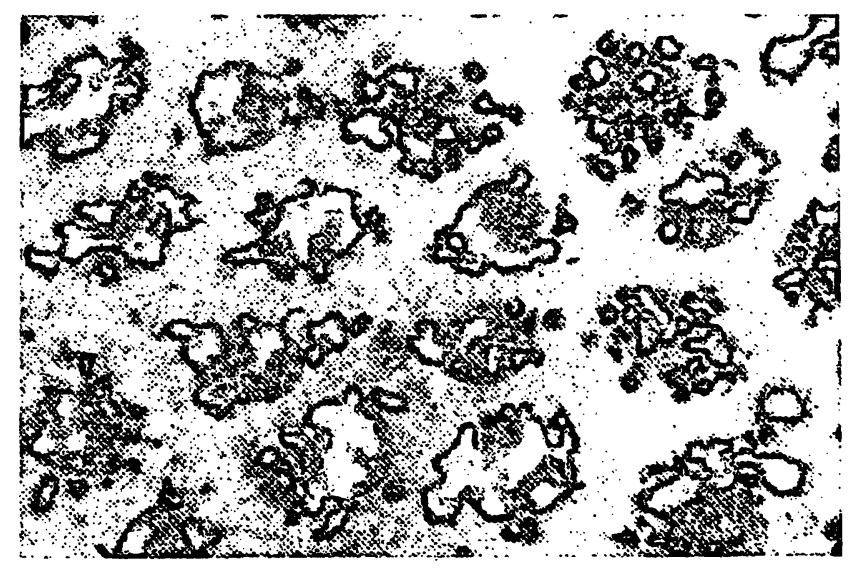

Abb. 1: Amnionepithel Mensch; Sektiomaterial: Häutchenpräparat nach Fixation in Formol-Calcium (n. Baker). Färbung der Lipide mit Sudan-Schwarz B. Endvergr. 800fach. 
Fruchtwasers in ihrer Substruktur nicht mit den Surfactantbildenden Einschlußkörpern der Alveolarzellen (Typ II) identisch sind, da ihnen die charakteristische Lamellenstruktur fehlt. Auch ist anzunehmen, daß der Surfactant nach dem Austritt aus der Alveolarzelle in einen solartigen Lösungszustand übergeht und morphologisch nicht mehr nachweisbar ist. Zur Absicherung der Frage nach der Identität der im Amnion und im Fruchtwasser nachweisbaren Lipide wurden die folgenden dünnschichtchromatographischen Untersuchungen angestellt.

\section{Material und Methode}

Von den frisch ausgestoßenen Sekundinae von 15 Graviditäten wurde das ",freie" Amnion abpräpariert und in physiologischer $\mathrm{NaCl}$-Lösung sorgfältig abgespült, um noch anhaftende Blutreste zu entfernen. Anschließend wurde das Material eingefroren und im Kryocut in $40 \mu \mathrm{m}$ dicke Schnitte zerkleinert. Dieser Gewebsbrei wurde dann nach Folch aufgearbeitet. In einem Chloroform-Methanol-Gemisch 2:1 (20fache Gewichtsmenge des Materials) wurden die Lipide extrahiert, der Rohextrakt durch ein fettfreies Filter filtriert, mit $1 / 5$ seines Volumens mit luftfreiem destilliertem Wasser versetzt und durchgeschüttelt. Nach 15 Minuten trennt sich das Gemisch in 2 Phasen. Die wässerige Phase wurde vorsichtig abgesaugt, die organische Phase mit den gelösten Lipiden im Rotationsverdampfer von $50 \mathrm{ml}$ auf $2 \mathrm{ml}$ eingeengt.

Nachweis der Lipide im Fruchtwasser: $10 \mathrm{ml}$ Fruchtwasser, das durch Amniocentese gewonnen worden war, wurde $5 \mathrm{~min}$ bei $3000 \mathrm{Upm}$ zentrifugiert und der Überstand dekantiert. Diese Oberphase wurde anschließend in der gleichen Weise wie oben zur Chromatographie vorbereitet.

Die chromatographische Auftrennung erfolgte auf Kieselgelplatten 60 F 254 (Merck) mit einer Schichtdicke von 0,25 mm. Am Start wurden von dem Konzentrat $20 \mu$ l aufgetragen. Der Referenzstandard bestand aus einem Gemisch von $L-\alpha-$ Lecithin, L- $\alpha$-Kephalin, Sphingomyelin, DL- $\alpha$-Phosphatidylserin und Phosphatidylinosit (alle Substanzen von der Fa. Serva) gelöst in Chloroform. Die aufgetragene Probenmenge betrug jeweils $20 \mu \mathrm{l}$. Als Laufmittel I wurde vorwiegend ein Chloroform:Methanol:Wasser-Gemisch im Verhältnis von 65:25:4 verwendet. Die Laufzeit betrug 2 Std. 30 Min. Außerdem wurde eine von Klima und Lindner (nicht publiziert). erarbeitete vereinfachte Modifikation des Laufmittels nach Kunz u. Mitarb. (1970) verwendet. Sie wird als Laufmittel II bezeichnet. Auf der getrockneten DC-Platte erfolgte die Darștellung der einzelnen Lipidfraktionen durch Joddämpfe, Dragendorff-Reagenz oder mit Bromthymolblau mit anschließender Entwicklung in $\mathrm{NH}_{3}$-Dämpfen.

In 10 Fällen erfolgte die Lipidbestimmung am Fruchtwasser und am Amnion derselben Schwangerschaft. Die Bestimmung der L/S-Ratio wurde nach den in der Literatur angegebenen Methoden vorgenommen.

\section{Ergebnisse}

\section{A. Lipide des Amnions}

Im Dünnschichtchromatogramm ließen sich auf Grund der mitgeführten Standards und der Berechnung der Rf-Werte mit dem Laufmittel I die unten angeführten Frakţionen unter-
Tab. 1:

\begin{tabular}{ll}
\hline Phospholipidfraktion & $\begin{array}{l}\text { Laufmittel I } \\
\text { Rf-Werte }\end{array}$ \\
\hline 1. Lysolecithin & 0,152 \\
2. Sphingomyelin & 0,168 \\
3. Phosphatidylserin & 0,182 \\
4. Phosphatidylinosit & 0,194 \\
5. Lecithin & 0,253 \\
6. Kephalin & 0,394 \\
7. Phosphatidyläthanol-Amin & 0,452 \\
8. Neutralfette u. freie Fettsäuren & 0,964 \\
\hline
\end{tabular}

scheiden, wobei die Fraktion 3 und 4 eine gemeinsame Bande bilden. Nahe der Laufmittelfront liegt eine gemeinsame Fraktion von Neutralfetten und Fettsäuren. Der Einsatz der Laufmittelmodifikation II brachte gegenüber dem Laufmittel I lediglich eine deutlichere Auftrennung nach 4 Stunden Laufzeit. Folgende Fraktionen konnten identifiziert werden (Abb. 2 und Tab. 1).

\section{B. Lipide des Fruchtwassers}

Bei unseren Untersuchungen unterscheiden sich im Dünnschichtchromatogramm die Lipidfraktionen des Fruchtwas-

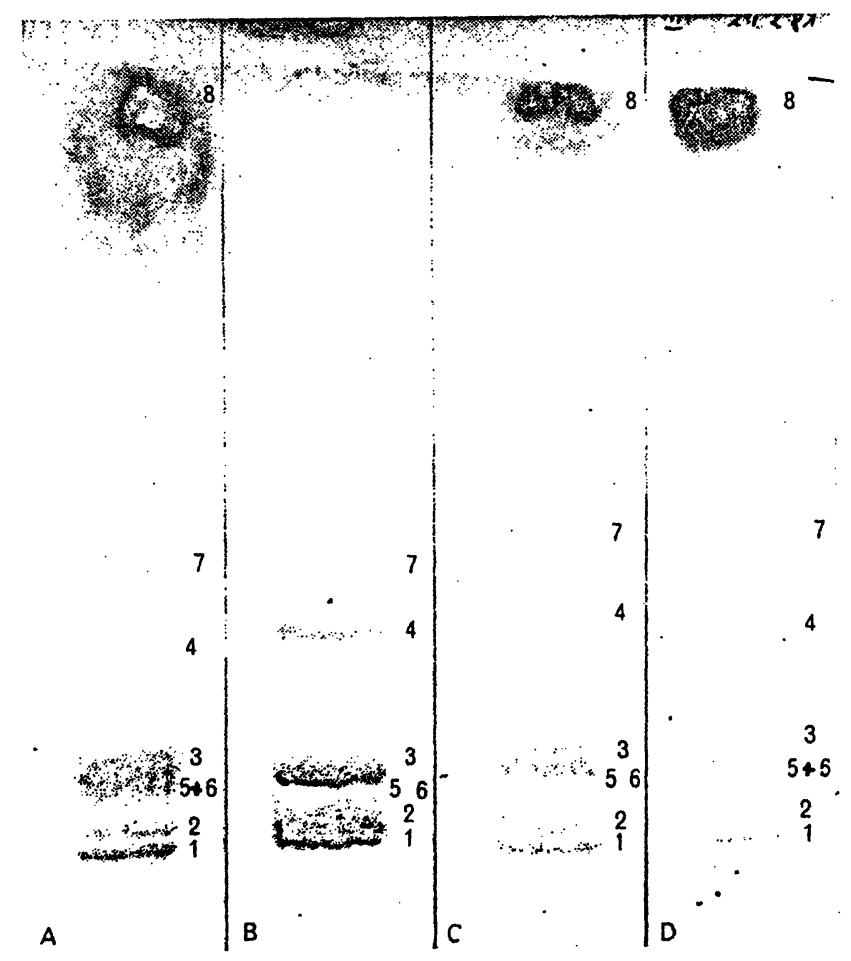

Abb. 2: Dünnschichtchromatogramm nach der oben aufgeführten Methode mit dem Laufmittel 1; Nachweis durch Joddämpfe. a, c und d Chromatogramme der Lipide aus dem Amnion von 3 verschiedenen Schwangerschaften; $b$ Referenzstandard. 1 Lysolecithin, 2 Sphingomyelin, 3 Phosphatidy/serin und 4 Phosphatidylinosit, 5 Lecithin, 6 Kephalin. 7 Phosphatidyläthanol-Amin, 8 Neutralfette und treie Fettsäuren (einschl. Cholesterinester). 
sers nicht von den in der Literatur angegebenen Worten. Durch die Anwendung des Laufmittels I wird eine Auftron. nung entsprechend don in dor Tobolle aulgefuhrton Fraktio. nen erreicht.

\section{Vergleichende Untersuchung an den Amnion- und Fruchtwasserlipiden der gleichen Schwangerschalt}

Diese Untersuchungen gingen von der Überlegung aus, daß bei Identität der Lipidtröpfchen im Amnionepithel und die Liposomen des Fruchtwassers auch die L/S-Ratio bei beiden, wenn sie von derselben Schwangerschaft stammen, gleich sein muB.

Das Ergebnis zeigte in allen untersuchten Fällen eine vollständige Übereinstimmung.

\section{Diskussion}

Nach den Ergebnissen der vorgelegten dünnschichtchromatographischen Analysen steht außer Frage, daß die Lipideinschlüsse des Amnions in Übereinstimmung mit den histochemischen Untersuchungen (4) außer Neutralfetten auch Phospholipide enthalten und zwar Lecithin und Sphingomyelin. Die Untersuchungen haben außerdem bestätigt, daß die Liposomen des Fruchtwassers aus Triglyzeriden, freien Fettsäuren und Phospholipiden bestehen. Die Lipidtröpfchen des Amnions und die Liposomen des Fruchtwassers sind also hinsichtlich ihrer Zusammensetzung identisch; ihre L/S-Ratio ist außerdem bei ein und derselben Schwangerschaft gleich. Wir erachten diesen Befund als eine weitere Bestätigung unserer Hypothese, nach der die Liposomen des Fruchtwassers aus den Amnionepithelzellen stammen. Die Tatsache, daß diese Herkunft und damit'auch die Herkunft der Phospholipide bisher nicht in Betracht gezogen wurde, hängt sicherlich mit der rein chemisch-präparativen Vorgangsweise zusammen, bei der das morphologische Substrat außer acht gelassen wird.

Die Frage, weshalb der Test und die Bestimmung der L/SRatio klinisch dennoch relevant ist und diese Methode einen brauchbaren Parameter zur Bestimmung der Lungenreife darstellt, können wir folgendermaßen erklären: Das Kompartiment Fett-Fruchtwasser-Eihäute ist eine entwicklungsgeschichtliche Einheit. Innerhalb dieser Einheit erfolgt die „Reifung", d. h. die Änderung der Lipidzusammensetzung in allen Anteilen gleichzeitig. Wie zuverlässig die Methode ist, kann ein Zufallsbefund bestätigen. Wir erhielten von einem Kreiskrankenhaus die Sekundinae eines Neugeborenen, das mit Atemnotsyndrom in die Universitätsklinik eingeliefert wordẹn war. Wir arbeiteten die Eihäute präparativ auf und fanden nach den üblichen Bestimmungsmethoden für Lecithin den Faktor 1,14 und für Sphingomyelin den Faktor 0,67. Die L/S-Ratio betrug 1,5, ein Wert, der sehr wohl auf ein mittelschweres Atemnotsyndrom schließen läßt. Bedauerlicherweise unterblieb in dem Kreiskrankenhaus die Bestimmung der L/S-Ratio im Fruchtwasser, da mit einer normalen Geburt gerechnet worden war.

Auch wenn wir der, wie wir meinen, gut begründeten Ansicht sind, daß die Liposomen des Fruchtwassers aus dem Amnion stammen, so können wir auf Grund der Untersuchungen von Setnikar et al. (8), von Boddy und Mantell (1) und auf Grund eigener Ergebnisse (7) nicht ausschließen, daß ein noch nicht bestimmbarer Anteil der Fruchtwasser-Phospholipide durch Expressionsbewegungen der fetalen Lunge ins Fruchtwasser gelangte und damit mit dem Surfactant identisch ist. Doch sind wir der Meinung. daß dieser Anteil im Vergleich zur Gesamtmenge der Phospholipide nur unwesentlich ist.

Schriltum.

1. BODOY. K.. MANTELL, C D. Obsenvations of fotal breathing movements transmited through maternal abdominal wall. Lancat 9. 1219 - 1220 (1972)

2. KUNZ, F.. MATT, G. HACKL. H. Plasma phospholipids in type IV nyperlipoproternemia Atherosclorosis 11. $265-270$ (1970)

3. SCHMIDT. W.: Det Feinbau der reiten menschichen Eihuute Z. Anat. Entwickl Gesch $119.203-222(1956)$

4. SCHMIDT, W.: Ubar den paraplacentaren, fruchtwassergebundenen Stofturanspon bein Menschen. I. Histochernische Untersuchung der in den Eihauien angereicherten Stoffe. Z Anat. Entwickl. Gesch. 124. 321 - 334 (1965).

5. SCHMIDT, W: Über den paraplacentaren, fruchtwassergebundenen Stofttransport beir Menschen. II. Nachwers der vom Amnion abgegebenen Lipide im Fruchtwasser und in Dünndarm des Keimes. Z. Anst. Entwickl. Gesch. 126, 276-288 (1967).

6. SCHMIDT, W., EBERHAGEN, D., SVEJCAR, J.: Uber den paraplacentaren, fruchtwas sergebundenen Siofftransport beim Menschen. III. Quantitative und qualitative Analyse de im Fruchtwasser enthaltenen Stoffe. Z. Anat. Entwickl. Gesch. 135, 210-221 (1971). 7. SCHMIDT, W. KLIMA, G.: Funktionen des Amnions im Kompartiment Fruchtwasser/Fel 2. Geburtsh. U. Perinat. 184, 248-254 (1980). 8. SETNIKAR, J., AGOSTINI, E., TAGLIETI, A.: The fetal lung, a source of amniotic fluid
Proc. Soc. Exp. Biol. (N.Y.) 10i, 842-845 (1969).

Anschrift der Verfasser:

Dr. Herbert Schwarzfurtner

Institut für Histologie und Embryologie

Dr. Günter Klima

Institut für Histologie und Embryologie

Müllerstraße 59

A-6010 Innsbruck 